\title{
ESTUDO CLÍNICO DA EFICÁCIA DO BLOQUEIO ANESTÉSICO RADICULAR TRANSFORAMINAL NO TRATAMENTO DA RADICULOPATIA LOMBAR
}

\author{
CLINICAL STUDY ON THE EFFICACY OF TRANSFORAMINAL RADICULAR BLOCK IN \\ LUMBAR RADICULOPATHY TREATMENT
}

ESTUDIO CLÍNICO DE LA EFICACIA DEL BLOQUEO ANESTÉSICO RADICULAR TRANSFORAMINAL EN EL TRATAMIENTO DE LA RADICULOPATIA LUMBAR

Fabiano Fonseca Rodrigues de Souza1, Rodrigo Junqueira Nicolau², Daiane Magioni Longo³, Luciano Miller Reis Rodrigues ${ }^{4}$

\begin{abstract}
RESUMO
Objetivo: O objetivo deste estudo é avaliar a eficácia da injeção transforaminal nos pacientes com dor radicular devido à hérnia de disco ou estenose foraminal lombar por meio de estudo prospectivo randomizado. Métodos: Foram avaliados 61 pacientes com quadro de radiculopatia nos membros inferiores. Esses pacientes foram divididos em dois grupos escolhidos aleatoriamente. Desses, 32 foram submetidos à injeção de corticosteroides e 29 foram submetidos à injeção salina. O período de acompanhamento foi de 12 meses. Para avaliar os resultados aplicamos a Escala Analógica Visual de Dor (EAV) e o Índice Oswestry (ODI). Resultados: Houve melhora significativa da intensidade de dor $(p<0,001)$ na primeira semana nas escalas EAV e ODI, em ambos os grupos estudados (corticosteroides e solução salina). Na observação após o terceiro mês, os dois grupos apresentaram resultados semelhantes. O uso do bloqueio transforaminal evidenciou-se um método eficaz para o tratamento da dor radicular aguda, e sem melhora da patologia se considerado a longo prazo. Conclusão: O bloqueio transforaminal é um excelente método com alta evidência para tratar a crise de dor radicular e ciática a curto prazo (menor que três meses), porém moderado a longo prazo (maior que seis meses).
\end{abstract}

Descritores: Dor; Corticosteroides; Ciática

\begin{abstract}
Objective: To assess the efficacy of corticosteroid injections in patients with sciatica due to lumbar disc herniation or lumbar foraminal stenosis by a prospective randomized study. Methods: There were analyzed 61 patients with sciatica due to lumbar disc degeneration. These patients were divided into two groups randomly chosen to radicular blocks with saline solution or corticosteroids. Thirty-one of these patients were submitted to corticosteroids radicular block and 29 patients were submitted to saline solution radicular block with a follow-up of 12 months. Outcomes were evaluated by visual analogue scale (VAS) and Oswestry disability index (ODI). Results: Statistical analysis showed improvement in pain reduction $(p<0.001)$ in the first week on ODI and VAS in the group with corticosteroid radicular block in comparison to the group with saline solution; however no statistical differences were observed after three months of follow-up. The transforaminal root block presents a good method for treatment of acute sciatica and showed no improvement in a long-term-follow-up. Conclusion: Transforaminal root block is an excellent method with high evidence to treat sciatica in short-term follow-up, but for long-term (more than six months) follow-up is just moderate.
\end{abstract}

Keywords: Pain; Costicosteroids; Sciatica.

\section{RESUMEN}

Objetivo: El presente estudio tuvo como objetivo evaluar la eficacia de la inyección transforaminal en pacientes con dolor radicular, debido a la hernia discal lumbar o estenosis foraminal, mediante un estudio prospectivo y aleatorizado. Métodos: Se analizó un total de 61 pacientes con cuadro de radiculopatía en miembros inferiores. Estos pacientes fueron divididos en dos grupos seleccionados al azar. De ellos, 31 fueron sometidos a inyecciones de corticoides y a 29 se les aplicaron inyecciones de suero fisiológico. El período de seguimiento fue de 12 meses. Para evaluar los resultados se les aplicó la Escala Visual Analógica del dolor (EAV) y el Indice de Oswestry (ODI, sigla en inglés). Resultados: Hubo una mejora significativa en la intensidad del dolor ( $p<0,001)$ en la primera semana según las escalas VAS y ODI, en ambos grupos estudiados (corticosteroides y solución salina). La observación después del tercer mes, en ambos grupos, mostró resultados similares. El uso del bloqueo transforaminal mostró un método eficaz para el tratamiento del dolor radicular agudo, y no hubo mejoría en la enfermedad en el largo plazo. Conclusión: El bloqueo transforaminal es un excelente método, con alta evidencia, para hacer frente a la crisis de dolor radicular y la ciática en el corto plazo (menos de 3 meses), pero tiene efecto moderado en el largo plazo (más de 6 meses).

Descriptores: Dolor; Corticoesteroides; Ciática.

1. Médico Residente do Grupo de Coluna da Faculdade de Medicina do ABC - São Paulo, SP, Brasil.

2. Médico Assistente do Grupo de Coluna da Faculdade de Medicina do ABC - São Paulo, SP, Brasil.

3. Psicóloga Hospitalar do Grupo de Coluna da Faculdade de Medicina do ABC - São Paulo, SP, Brasil.

4. Chefe do Grupo de Coluna da Faculdade de Medicina do ABC - São Paulo, SP, Brasil.

Trabalho realizado no Departamento do Aparelho Locomotor da Faculdade de Medicina do ABC

Correspondência: Rua Henrique Calderazzo, 321 - 5ªndar (Centro de Estudos) - CEP: 09190-615 - Santo André, SP, Brasil. Email: dr.fabianosouza@ hotmail.com

Recebido em 30/06/2010, aceito em 09/11/2010. 


\section{INTRODUÇÃO}

Injeções epidurais de anestésicos locais ou corticosteróides estão sendo atualmente utilizadas no tratamento das dores radiculares e lombares, principalmente para o tratamento da hérnia de disco, estenose de canal, estenose foraminal, artrose facetária e como diagnóstico diferencial do nível acometido na compressão radicular $^{1,2}$. A taxa de sucesso varia entre $20 \%$ e $90 \%$, porém com período de duração muito variável ${ }^{3}$. As infiltrações epidurais não alteram o curso do processo evolutivo da doença, mas podem oferecer um alívio de dor nos pacientes selecionados. A sua indicação compreende patologias na região cervical, torácica, lombar e sacral.

O primeiro registro de uso de corticóide por via epidural foi feito por Robecchi e Capra ${ }^{1}$ com uso de hidrocortisona. Os corticosteróides exercem sua ação analgésica antiinflamatória, impedindo a liberação de ácido araquidônico da membrana das células lesadas. Essa medicação age na: diminuição no edema, depósito de fibrina, dilatação capilar, migração de leucócitos, proliferação de capilares e de fibroblastos, deposição de colágeno e cicatrização ${ }^{4,5}$. Estudos também demonstram que os corticosteróides reduzem a hiperexcitabilidade da célula nervosa por agir diretamente na condução da membrana celular²; deste modo, há redução do edema das raízes nervosas e tecidos adjacentes. Pode-se dizer que o efeito curativo da injeção peridural de corticosteróide não resulta de efeito de volume para lise de aderências, mas do próprio efeito antiinflamatório ${ }^{1}$.

Com a associação de contrastes radiopacos nas infiltrações transforaminais lombares guiadas por radioscopia obteve-se maior precisão na localização da raíz nervosa. Desta forma, com menor volume de anestésico, conseguiu-se uma maior concentração no local desejado5,6. No entanto, apesar do desenvolvimento da técnica, existem estudos que não defendem a melhora clínica para a utilização da infiltração de corticóides como modalidade terapêutica ${ }^{7,8}$. Como nos primeiros estudos utilizados por Cuckler et al. ${ }^{9}$, que comparou a injeção de anestésicos locais com a injeção de anti-inflamatórios, não evidenciando superioridade da injeção de corticosteróides em relação ao anestésico local.

Os bloqueios radiculares transforaminais lombares guiados por radioscopia são procedimentos amplamente empregados como técnica de terapia intervencionista para o tratamento de dor. Esta terapia fornece alívio quase imediato da dor e possibilidade de tratamento para a dor de causa discogênica nas radiculopatias lombares, uma vez que este alívio auxilia nas repostas aos métodos de tratamento de reabilitação ${ }^{10}$

Este estudo tem como objetivo avaliar a eficácia das injeções epidurais com o uso de corticoteróides no tratamento da radiculopatia lombar, comparando o seu uso com o da solução salina (soro fisiológico 0,9\%)

\section{MÉTODOS}

Foram avaliados 61 pacientes com dor radicular em membros inferiores, no período de fevereiro de 2008 a outubro de 2009. Todos realizaram ressonância magnética lombar, e o nível acometido variou de L3-L4 até L5-S1.

Os pacientes foram divididos em dois grupos escolhidos aleatoriamente. $\mathrm{O}$ grupo A era constituído de 32 pacientes submetidos à injeções com anestésico e corticosteróides; e o grupo B constituía-se de 29 pacientes submetidos à injeção de solução de soro fisiológico 0,9\%. O trabalho foi aprovado pelo comitê de ética com o número 262/2008.

O procedimento foi realizado somente por dois médicos especialistas em cirurgia da coluna, com conhecimento prévio da solução que estavam utilizando no procedimento. A psicóloga do grupo da coluna aplicava os questionários não tendo conhecimento prévio sobre a que grupo o paciente pertencia. Durante o período de seguimento os pacientes foram acompanhados pela equipe de fisioterapia e reabilitação do Hospital.

Os critérios de inclusão foram: 1) paciente com sintomas de dor radicular em membros inferiores, maior que dor lombar, persistente por mais de 6 semanas; 2) sinais clínicos de radiculopatia com testes de elevação do membro inferior e manobra de Laségue positivo; 3) exame de ressonância magnética que apresentava discopatia lombar e compressão em nível do forame intervertebral compatível com exame clínico; 4) idade mínima de 18 anos; 5) ausência de instabilidade segmentar demonstrada em radiografia dinâmica.

O critérios de exclusão foram pacientes com coagulopatias, infecção de pele, grávidas, espondiloartropatia, psoríase, doença inflamatória intestinal, artrite séptica, estenose de canal medular ou do recesso lateral com sintomas de claudicação neurogênica e cirurgia prévia lombar. Todos os pacientes assinaram Termo de Consentimento Informado Livre Esclarecido, contendo os riscos e benefícios da terapia instituída.

$\mathrm{Na}$ avaliação do paciente foi aplicada a escala visual analógica da dor (EAV) e o questionário de incapacidade Oswestry (ODI), sendo todos os questionários aplicados pela mesma pessoa.

$\mathrm{Na}$ técnica realizada, o paciente era posicionado em decúbito ventral horizontal, com apoio (travesseiro) sob abdome, quadris e joelhos semi-fletidos a aproximadamente 30 graus. Os parâmetros vitais eram monitorizados. Após assepsia e colocação de campos estéreis eram realizados exames de imagem com radioscopia. Primeiramente, o nível a ser infiltrado era determinado com realização da incidência ântero-posterior absoluta. De acordo com o nível, muitas vezes foi necessário realizar a incidência descrita por Ferguson, de angulação cranial da radioscopia, principalmente no nível L5-S1. Uma vez que se visibilizava a placa terminal como uma linha única, sem duplo contorno, o radioscópio era rodado para o lado acometido, gerando uma imagem oblíqua do lado invertido. Geralmente entre 20 e 25 graus eram suficientes para se obter uma correta incidência, determinando uma correta região anatômica dos pedículos e facetas articulares. O posicionamento do paciente era mantido e a angulação no plano crânio-caudal com relação à angulação era anotado pelo técnico em radioscopia. Um fórceps Kelly reto era apoiado sob a região lombar do paciente para estabelecer o alvo exato, utilizando-se, assim, a ponta do instrumento que era mobilizada até coincidir exatamente com o alvo da imagem correspondente à região inferior do pedículo com leve desvio medial. Uma infiltração local do alvo com lidocaína $2 \%$ era realizada, então a agulha espinhal 22Gauges e com 3,5in de comprimento com ponta de Quincke era introduzida. Em pacientes maiores ou obesos, uma agulha mais longa era necessária. A agulha era então introduzida, seguindo a técnica coaxial, e observada como um único ponto ao seguir o mesmo trajeto de incidência na radioscopia (Figura 1A).

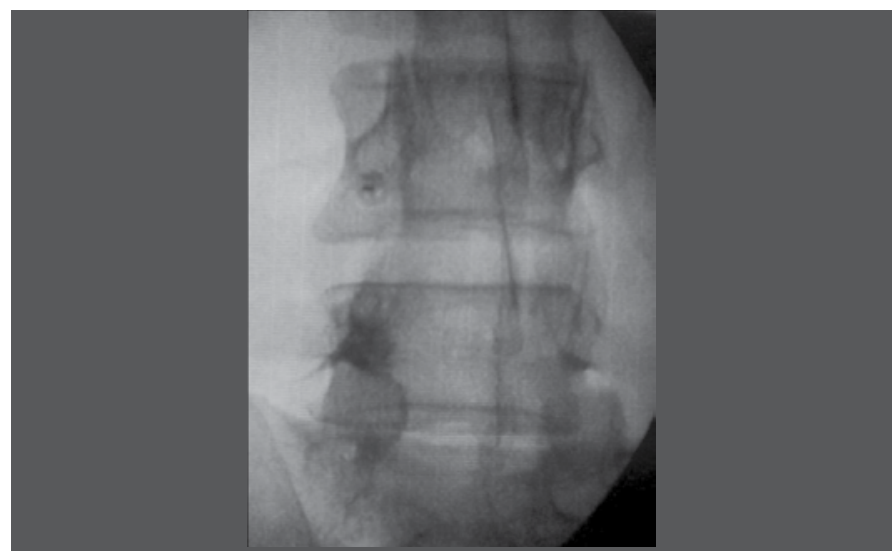

Figura 1A. Radioscopia ântero-posterior (Fergusson) com obliqüidade de 20 graus mostrando agulha com ponto único inferior e medial ao pedículo de $L 4$ a direita.

Conforme a agulha era introduzida a posição da radioscopia era alterada para fornecer a incidência de perfil absoluto, então a profundidade da agulha era controlada, principalmente na região do forame intervertebral. Próximo à porção inferior do pedículo, o radioscópio retornava para a posição oblíqua ou ântero-posterior absoluta. Uma pequena extensão era acoplada à agulha, a fim de 
evitar sobreposição da mão na imagem. Injetava-se o contraste não iônico (p.ex.: lohexol 300mg/ml) sob visão contínua da radioscopia para acompanhar a distribuição do contraste no espaço epidural e proteger de sua introdução no meio intravascular (Figuras 1B e 1C). Uma vez obtida uma distribuição adequada, injetava-se uma solução que podia ser salina (soro fisiológico 0,9\%) ou composta de anestésico e corticosteróides (40mg de metilpredinisolona e 2ml de bupivacaina a 0,25\%). No caso do forame de L5-S1, uma visão ântero-posterior era realizada e a agulha posicionada no quadrante superior externo do forame, com a técnica coaxial, assim o perfil era acompanhado até atingir a posição do forame. Após o procedimento, o paciente era encaminhado para recuperação pós-anestésica e permanecia em observação por aproximadamente 1 hora. Nenhuma medicação era prescrita depois do procedimento.

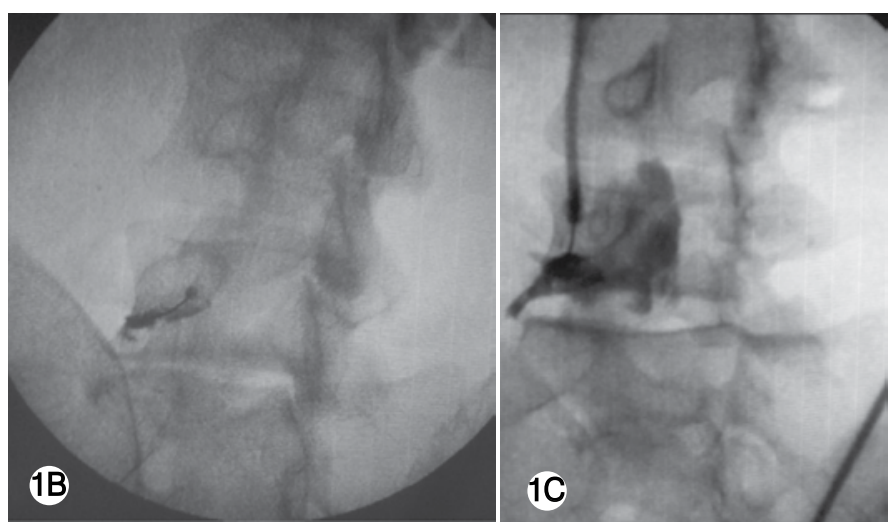

Figuras 1B e 1C. Radioscopia com injeção de contraste não iônico seguindo o trajeto da raiz de $L 4$ a direita.

Os questionários EAV e ODI foram aplicados antes e 1 hora após o procedimento. As avaliações seguintes foram realizadas com 1 semana, 2 semanas, 1 mês, 3 meses, 6 meses e 1 ano, seguindo o mesmo método de avaliação. Na última avaliação era perguntado se fariam ou não o procedimento novamente, caso necessário, e se se encontravam melhor do que antes. Uma redução de $10 \%$ no ODI foi considerada uma mudança clinicamente significativa e uma mudança de $20 \mathrm{~mm}$ na escala analógica da dor também foi considerado clinicamente significativo. No $1^{\circ}$ ano de acompanhamento o resultado foi avaliado em termos da necessidade de cirurgia ou necessidade de reintervenção por meio de bloqueio transforaminal. A necessidade de nova intervenção baseou-se em dor na perna residual significativo.

Os dados foram submetidos à análise univariada, utilizando amostras independentes, teste $t$ de Student, de ensaio ou teste exato de Fisher. Os resultados foram considerados estatisticamente significativos quando os valores $P$ foram inferiores a 0,05 . Os dados foram analisados usando o software SPSS 12.0. O Teste de Mann-Whitney foi aplicado com o intuito de verificarmos possíveis diferenças entre ambos os grupos estudados, para as variáveis de interesse.

\section{RESULTADOS}

Dos 61 pacientes incluídos neste estudo 4 apresentaram cefaléia após o procedimento e 1 paciente apresentou diminuição de força no dermátomo correspondente à raíz infiltrada, mas com recuperação após 4 horas. Nenhum dos pacientes apresentou cefaléia persistente ou sintomas que sugerissem lesão dural.

Dos pacientes estudados, 34 eram mulheres e 27 homens. A idade variou entre 26,2 anos e o de maior idade 63,1 anos com uma média de idade de 41,06 anos. A duração média desde o inicio dos sintomas foi de 11,7 meses \pm 6,7 meses.

No seguimento pós-bloqueio, 7 pacientes foram indicados para a cirurgia antes de três meses de acompanhamento. Dois pacientes não apresentaram melhora em seis semanas de acompanhamento. Dois pacientes perderam seguimento com três meses. Portanto, em 12 semanas de seguimento, os dados foram disponíveis para 50 pacientes. Em seis meses de seguimento, outros 7 pacientes haviam descontinuado o acompanhamento ou realizado cirurgia por dor intratável, sendo que os dados finais estavam disponíveis para 43 pacientes. Não houve complicação significativa em nenhum dos pacientes estudados.

Durante o estudo, a maior alteração encontrada foi no EAV após 1 semana de procedimento no grupo $A$ em que se utilizou solução de corticóide $(P<0,001)$ (Figura 2$)$, sendo que o grupo de solução com corticosteróides teve uma redução média de 3,4 pontos enquanto o grupo de solução salina ficou em aproximadamente em 1 ponto; obteve-se também uma diferença estatisticamente significativa no mesmo grupo I em relação ao questionário ODI após 2 e 3 meses do procedimento $(P<0,005)$ (Figura 3$)$; sendo uma variável media de 10 pontos em relação aos resultados obtidos antes do procedimento. Neste mesmo grupo A houve uma diferença em média de 5 pontos do ODI em relação aos pacientes que realizaram injeção de solução salina (Tabela 1) para o mesmo período.

Houve uma variação estatística significante nos pacientes que utilizaram corticóide e anestésico a curto prazo em relação a dor e também uma melhora significante a longo prazo em relação ao questionário Oswestry.

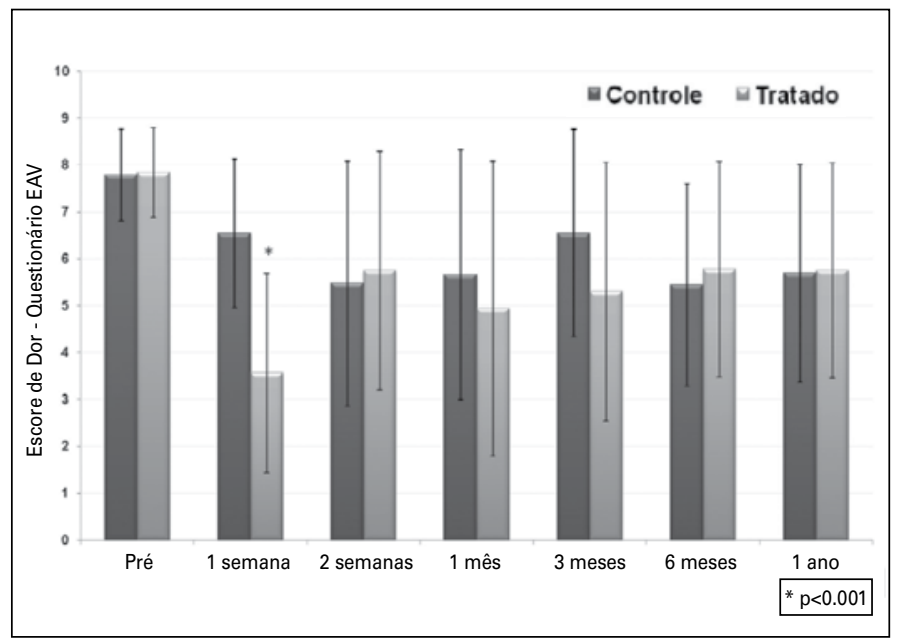

Figura 2. Avaliação da dor com questionário EAV pós bloqueio após bloqueio com corticóide e com solução salina com seguimento até 1 ano.

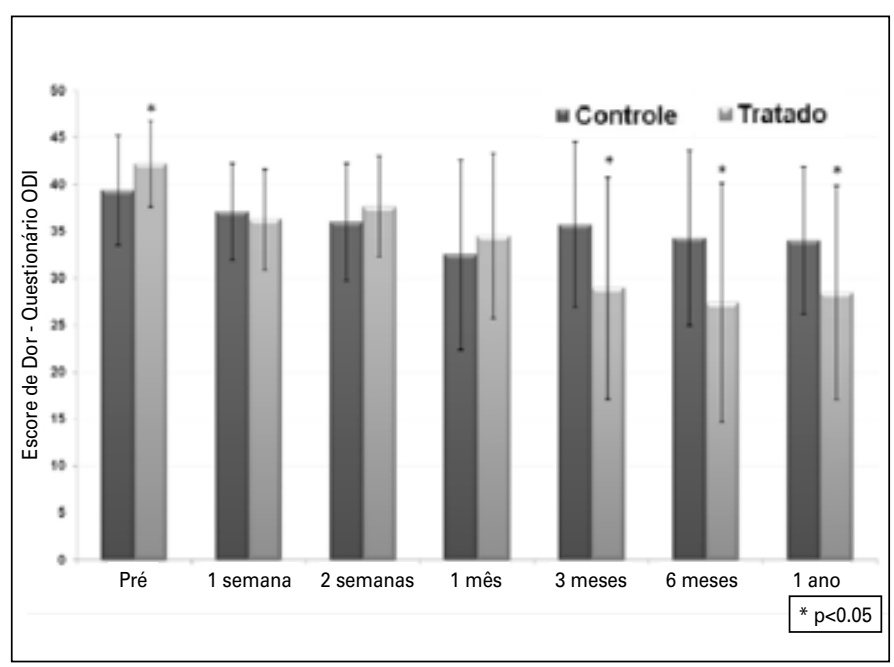

Figura 3. Avaliação da dor através do questionário de índice de incapacidade de Oswestry após bloqueio com corticóide e com solução salina com seguimento até 1 ano 
Tabela 1 - Avaliação das variáveis: idade, duração da dor, avaliação da dor pré-tratamento e pós-imediato, 1 semana, 2 semanas, 1 mês, 3 meses, 6 meses e 1 ano com injeções de corticóide e solução salina através dos questionários EAV e Oswestry.

\begin{tabular}{|c|c|c|c|c|c|c|c|c|c|c|}
\hline Variável & Grupo & $\mathbf{N}$ & Média & Desvio-padrão & Mínimo & Máximo & Percentil 25 & Mediana & Percentil 75 & Sig. (p) \\
\hline \multirow{3}{*}{ Idade } & A & 32 & 41,06 & 11,21 & 26,00 & 63,00 & 32,00 & 38,50 & 50,00 & \multirow{2}{*}{0,563} \\
\hline & $B$ & 29 & 42,97 & 11,76 & 26,00 & 63,00 & 33,00 & 48,00 & 54,75 & \\
\hline & Total & 61 & 41,97 & 11,42 & 26,00 & 63,00 & 32,25 & 40,50 & 54,00 & \\
\hline \multirow{3}{*}{ Sintomas (meses) } & A & 32 & 7,63 & 3,71 & 3,00 & 16,00 & 4,25 & 6,50 & 10,75 & \multirow{2}{*}{0,322} \\
\hline & $\mathrm{B}$ & 29 & 6,69 & 3,32 & 3,00 & 12,00 & 4,25 & 7,00 & 12,00 & \\
\hline & Total & 61 & 7,18 & 3,53 & 3,00 & 16,00 & 4,25 & 6,50 & 11,00 & \\
\hline \multirow{3}{*}{ EAV PRE } & A & 32 & 7,84 & 0,95 & 6,00 & 9,00 & 7,00 & 8,00 & 9,00 & \multirow{2}{*}{0,838} \\
\hline & B & 29 & 7,79 & 0,98 & 6,00 & 9,00 & 7,00 & 8,00 & 8,00 & \\
\hline & Total & 61 & 7,82 & 0,96 & 6,00 & 9,00 & 7,00 & 8,00 & 8,75 & \\
\hline \multirow{3}{*}{ EAV pos } & A & 32 & 3,69 & 1,40 & 2,00 & 6,00 & 2,00 & 4,00 & 5,00 & \multirow{2}{*}{$<0,001$} \\
\hline & B & 29 & 6,48 & 1,18 & 4,00 & 8,00 & 5,25 & 7,00 & 7,00 & \\
\hline & Total & 61 & 5,02 & 1,91 & 2,00 & 8,00 & 3,00 & 5,00 & 6,00 & \\
\hline \multirow{3}{*}{ EAV 1 sem pos } & $\mathrm{A}$ & 32 & 3,56 & 2,12 & 1,00 & 8,00 & 2,00 & 3,00 & 4,75 & \multirow{2}{*}{$<0,001$} \\
\hline & B & 29 & 6,55 & 1,59 & 2,00 & 8,00 & 5,50 & 7,00 & 7,00 & \\
\hline & Total & 61 & 4,98 & 2,41 & 1,00 & 8,00 & 2,00 & 4,50 & 7,00 & \\
\hline \multirow{3}{*}{ EAV 2 sem } & $\mathrm{A}$ & 32 & 5,75 & 2,54 & 0,00 & 9,00 & 4,00 & 6,00 & 8,00 & \multirow{2}{*}{0,612} \\
\hline & $B$ & 29 & 5,48 & 2,61 & 1,00 & 9,00 & 2,25 & 6,00 & 8,00 & \\
\hline & Total & 61 & 5,62 & 2,56 & 0,00 & 9,00 & 3,25 & 6,00 & 8,00 & \\
\hline \multirow{3}{*}{ EAV 1 mes } & $A$ & 28 & 4,94 & 3,15 & 0,00 & 9,00 & 2,00 & 6,00 & 8,00 & \multirow{2}{*}{0,320} \\
\hline & $\mathrm{B}$ & 27 & 5,66 & 2,66 & 1,00 & 9,00 & 3,00 & 6,50 & 8,00 & \\
\hline & Total & 55 & 5,28 & 2,93 & 0,00 & 9,00 & 3,00 & 6,00 & 8,00 & \\
\hline \multirow{3}{*}{ EAV 3 meses } & A & 27 & 5,31 & 2,76 & 0,00 & 8,00 & 2,00 & 6,50 & 8,00 & \multirow{2}{*}{0,073} \\
\hline & $\mathrm{B}$ & 23 & 6,55 & 2,21 & 0,00 & 9,00 & 5,00 & 6,50 & 8,00 & \\
\hline & Total & 50 & 5,90 & 2,57 & 0,00 & 9,00 & 4,00 & 6,50 & 8,00 & \\
\hline \multirow{3}{*}{ EAV 6 meses } & $\mathrm{A}$ & 25 & 5,78 & 2,30 & 0,00 & 8,00 & 5,00 & 7,00 & 7,00 & \multirow{2}{*}{0,310} \\
\hline & $\mathrm{B}$ & 22 & 5,45 & 2,16 & 1,00 & 9,00 & 4,25 & 6,00 & 7,00 & \\
\hline & Total & 47 & 5,62 & 2,22 & 0,00 & 9,00 & 5,00 & 7,00 & 7,00 & \\
\hline & A & 23 & 5,75 & 2,29 & 1,00 & 8,00 & 3,50 & 7,00 & 8,00 & 0866 \\
\hline EAV 1 ano & $B$ & 20 & 5,69 & 2,32 & 2,00 & 9,00 & 5,00 & 6,00 & 8,00 & 0,000 \\
\hline & Total & 43 & 5,72 & 2,28 & 1,00 & 9,00 & 5,00 & 6,00 & 8,00 & \\
\hline & A & 32 & 42,19 & 4,58 & 30,00 & 52,00 & 38,00 & 42,00 & 46,00 & 0008 \\
\hline ODI PRE & B & 29 & 39,38 & 5,83 & 28,00 & 58,00 & 38,00 & 40,00 & 42,00 &, 000 \\
\hline & Total & 61 & 40,85 & 5,36 & 28,00 & 58,00 & 38,00 & 42,00 & 44,00 & \\
\hline & A & 32 & 36,25 & 5,39 & 22,00 & 42,00 & 34,00 & 38,00 & 41,50 & 0.477 \\
\hline ODI 1 sem pos & $\mathrm{B}$ & 29 & 37,10 & 5,17 & 22,00 & 42,00 & 36,00 & 38,00 & 42,00 & $0,4 / 7$ \\
\hline & Total & 61 & 36,66 & 5,26 & 22,00 & 42,00 & 34,00 & 38,00 & 42,00 & \\
\hline & $\mathrm{A}$ & 32 & 37,63 & 5,41 & 22,00 & 44,00 & 36,00 & 38,00 & 42,00 & 0310 \\
\hline ODI 2sem & $B$ & 29 & 36,00 & 6,28 & 22,00 & 44,00 & 30,00 & 37,00 & 43,50 & טוכונ \\
\hline & Total & 61 & 36,85 & 5,85 & 22,00 & 44,00 & 34,00 & 38,00 & 42,00 & \\
\hline & $\mathrm{A}$ & 28 & 34,50 & 8,78 & 10,00 & 46,00 & 30,00 & 34,00 & 42,00 & 0494 \\
\hline ODI 1 mes & $B$ & 27 & 32,48 & 10,14 & 10,00 & 44,00 & 27,00 & 36,00 & 42,00 & 0,494 \\
\hline & Total & 55 & 33,54 & 9,43 & 10,00 & 46,00 & 30,00 & 34,00 & 42,00 & \\
\hline & A & 27 & 28,94 & 11,81 & 10,00 & 44,00 & 20,00 & 31,00 & 39,50 & 0025 \\
\hline ODI 3 meses & $B$ & 23 & 35,72 & 8,80 & 10,00 & 44,00 & 30,00 & 39,00 & 42,00 & $0,0<5$ \\
\hline & Total & 50 & 32,16 & 10,94 & 10,00 & 44,00 & 22,50 & 35,00 & 42,00 & \\
\hline & A & 25 & 27,38 & 12,72 & 8,00 & 42,00 & 10,00 & 30,00 & 38,00 & 0038 \\
\hline ODI 6 meses & $B$ & 22 & 34,30 & 9,37 & 10,00 & 44,00 & 30,00 & 38,00 & 41,50 & 0,038 \\
\hline & Total & 47 & 30,04 & 11,94 & 8,00 & 44,00 & 26,00 & 34,00 & 38,00 & \\
\hline & A & 23 & 28,44 & 11,41 & 10,00 & 42,00 & 19,50 & 30,00 & 38,00 & 0059 \\
\hline ODI 1 ano & $B$ & 20 & 34,00 & 7,84 & 14,00 & 44,00 & 30,00 & 38,00 & 40,00 &, 000 \\
\hline & Total & 43 & 31,08 & 10,19 & 10,00 & 44,00 & 26,00 & 34,00 & 38,00 & \\
\hline
\end{tabular}




\section{DISCUSSÃO}

Este estudo demonstrou que a infiltração com corticosteróides associada a anestésicos locais possui um efeito agudo no tratamento da dor radicular, com melhora e diminuição das escalas de dor quando comparados com a injeção de solução salina.

A dor provocada por uma hérnia de disco, além do efeito mecânico compressivo, ocorre uma irritação química da raiz nervosa pela alta concentração de nor-receptores e mediadores inflamatórios próximos da raiz nervosa ${ }^{8,10}$. Desta forma, acredita-se que os corticosteróides podem reduzir o componente inflamatório da raiz nervosa ${ }^{11,12}$. O mecanismo exato de ação dos corticosteróides na dor radicular é desconhecido, mas sabe-se que, juntamente com compressão mecânica das raízes nervosas, a dor ciática é causada por disparo ectópico de mediadores químicos diversos. Estas incluem prostaglandina $\mathrm{E}$ e um mediador químico da inflamação ${ }^{13,14}$.

Cuckler et al. ${ }^{9}$ demonstraram que apesar do grande benefício proporcionado pelo corticóide, este não produzia qualquer benefício adicional comparado com a anestesia local. Nosso estudo, apesar de ter sido realizado com a injeção de anestésico local associado a corticóide, permite inferir que, pelo curto prazo de ação analgésica observada, a ação antiinflamatória foi limitada; e que a melhora de dor por um curto período pode estar relacionada à ação do anestésico local, fato este que necessita de novos estudos para avaliação da resposta clínica em relação ao tempo diante dessas duas situações.

Karpinnen et al. ${ }^{15}$, em seu artigo, mostraram que a infiltração peri-radicular de corticóide produz um benefício a curto prazo em termos de melhora da ciátia. Este estudo constatou que houve uma melhora na dor em 2 semanas de $45 \%$ no grupo esteróide quando comparados com $24 \%$ no grupo de solução salina; mas em 3 meses, 6 meses e 1 ano após a injeção não houve diferença entre os grupos analisados. Resultados estes semelhantes aos obtidos em nosso estudo. Isto muito provavelmente se deve pela nova formação de mediadores químicos inflamatórios no local de contato do disco com a raiz nervosa.

Uma das possibilidades da infiltração, utilizando-se a via transforaminal, apresentar um maior período de duração do bloqueio, pode ser por permitir o depósito direto da medicação no local exato da patologia. Além disto, o potente efeito anti-inflamatório dos esteróides pode levar a diminuição dos mediadores inflamatórios da região, tais como interleucina-1, fator de necrose tumoral e fosfolipase-A2 ${ }^{16}$. Assim como citado pelo estudo de Nicolau e Rodrigues $^{17}$, este efeito antiinflamatório realizado pela injeção de corticóide pela via transforaminal para o alivio da dor é um método seguro para o tratamento da ciatalgia.

O estudo realizado por Thomas et al. ${ }^{18}$ demonstrou que a injeção transforaminal de corticosteróide com controle radioscópico prevê melhor alívio da dor (EAV), e apresenta maior eficácia quando comparado a injeção intraespinhosa. Isso justifica a técnica usada em nosso estudo com a segmentação da raiz nervosa selecionada para injeção da solução sob fluoroscopia.

Tafazal et al. ${ }^{16}$ comparou o tratamento conservador para dor radicular demonstrando que quando se compara a injeção peridural de corticosteróide com o anestésico local não há diferença a longo prazo nos resultados clínicos, e nenhuma diferença significativa em relação ao retorno ao trabalho durante o acompanhamento; fato também encontrado em nosso estudo.

Um estudo realizado por Carette et al. ${ }^{3}$ também encontrou que o corticosteróide epidural fornece apenas benefícios de curto prazo em pacientes com dor ciática causado por um disco prolapsado. Este estudo comparou o corticóide epidural com placebo de solução salina e em seis semanas os autores descobriram que a melhora na dor nas pernas foi maior no grupo da anestesia peridural de corticosteróides. Em três meses, não houve diferença significativa no resultado entre os grupos. Em 1 ano de seguimento, a quantidade de indicação cirúrgica também foi similar em ambos os grupos (25,8\% no grupo do corticosteróide vs 24,8\% no grupo placebo). Estes dados confirmam os nossos resultados de que os corticosteróides não oferecem benefício a longo prazo para evitar possíveis candidatos a uma intervenção cirúrgica, mas proporcionam um bom alívio na dor possibilitando a esses pacientes diminuir o uso de medicação e suportarem a evolução da história natural da doença ${ }^{19,20}$.

\section{CONCLUSÃO}

A infiltração peri-radicular de corticosteróides para a ciática não fornece benefício adicional a longo prazo quando comparada com a injeção de solução salina.

O procedimento é eficaz em termos de alívio da dor a curto prazo em pacientes com diagnóstico de radiculopatia lombar. Coloca-se como um método seguro e eficaz no tratamento agudo da dor ciática.

\section{REFERÊNCIAS}

1. Robecchi A, Capra R. L'idrocortisone (composto F): prime espe- rienze cliniche in campo reumatologico. Minerva Med. 1952:98:1259.

2. Tonkovich-Quaranta LA, Winkler SR. Use of epidural corticosteroids in low backpain. Ann Pharmacother. 2000;34(10):1165-72.

3. Carette $S$, Leclaire $R$, Marcoux S, Morin F Blaise GA St-Pierre A, et al. Epidural corticosteroid injections for sciatica due to herniated nucleus pulposus. N Engl J Med. 1997;336(23):1634-40.

4. Cannon DT, Aprill CN. Lumbosacral epidural steroid injections. Arch Phys Med Rehabil. 2000:81(3Suppl1):S87-98.

5. Spaccarelli KC. Lumbar and caudal epidural corticosteroid injections. Mayo Clin Proc. 1996;71(2):169-78

6. Airaksinen O, Brox Jl, Cedraschi C Hildebrandt J Klaber-Moffett J, Kovacs F et al. COST B13 Working Group on Guidelines for Chronic Low Back Pain. Chapter 4. European guidelines for the management of chronic nonspecific low back pain. Eur Spine J. 2006:15(Suppl 2):S192-300.

7. Manchikanti L, Helm S, Singh V, Benyamin RM, Datta S, Hayek SM, et al. ASIPP. An algorithmic approach for clinical management of chronic spinal pain. Pain Physician. 2009:12(4):E225-64.

8. Rosen CD, Kahanovitz N, Bernstein R, Viola K. A retrospective analysis of the efficacy of epidural steroid injections. Clin Orthop Relat Res. 1988;(228):270.

9. Cuckler JM, Bernini PA, Wiesel SW, Booth RE Jr, Rothman RH, Pickens GT. The use of epidural steroids in the treatment of lumbar radicular pain. A prospective, randomized, double-blind study. J Bone Joint Surg Am. 1985;67(1):63-6.

10. Nelson DA, Landau WM. Intraspinal steroids: history, efficacy, accidentality, and controversy with review of United States Food and Drug Administration reports. J Neurol Neurosurg Psychiatry. 2001;70(4):433-43.

11. Manchikanti L, Boswell MV, Datta S, Fellows B, Abdi S, Singh V, et al. ASIPP. Comprehen-

sive review of therapeutic interventions in managing chronic spinal pain. Pain Physician. 2009:12(4):E123-98.

12. Buenaventura RM, Datta S, Abdi S, Smith HS. Systematic review of therapeutic lumbar transforaminal epidural steroid injections. Pain Physician. 2009:12(1):233-51.

13. Saal JA, Saal JS, Herzog RJ. The natural history of lumbar intervertebral disc extrusions treated nonoperatively. Spine (Phila Pa 1976). 1990;15(7):683-6.

14. Koc Z, Ozcakir S, Sivrioglu K, Gurbet A, Kucukoglu S. Effectiveness of physical therapy and epidural steroid injections in lumbar spinal stenosis. Spine (Phila Pa 1976). 2009:34(10):985-9.

15. Karppinen J, Malmivaara A, Kurunlahti M, Kyllönen E, Pienimäki T, Nieminen P,et al. Periradicular infiltration for sciatica: a randomized controlled trial. Spine (Phila Pa 1976). 2001 May 1:26(9):1059-67.

16. Tafazal $S, N g ~ L$, Chaudhary N, Sell P. Corticosteroids in peri-radicular infiltration for radicular pain: a randomised double blind controlled trial. One year results and subgroup analysis. Eur Spine J. 2009:18(8):1220-5

17. Nicolau RJ., Rodrigues LM. Resultados Terapeuticos das infiltrações epidurais, pela via transforaminal, guiadas por radioscopia em pacientes com radiculopatia lombosacral. Rev Bras Med. 2009; 66(12):27-31.

18. Thomas E, Cyteval C, Abiad L, Picot MC, Taourel P, Blotman F. Efficacy of transforaminal versus interspinous corticosteroid injectionin discal radiculalgia - a prospective, randomised, double-blind study. Clin Rheumatol. 2003:22(4-5):299-304

19. Lee JH, An JH, Lee SH. Comparison of the effectiveness of interlaminar and bilateral transforaminal epidural steroid injections in treatment of patients with lumbosacral disc herniation and spinal stenosis. Clin J Pain. 2009:25(3):206-10.

20. Riew KD, Yin Y, Gilula L, Bridwell KH, Lenke LG, Lauryssen $C$, et al. The effect of nerve-root injections on the need for operative treatment of lumbar radicular pain. A prospective, randomized, controlled, double-blind study. J Bone Joint Surg Am. 2000;82(11):1589-93. 